\section{Chat capability now live}

Simple, secure and reliable messaging is something that many of us use in our everyday lives. It might be talking to family, groups of friends, work colleagues or even your child's school through a PC, tablet or mobile device - not to mention Live Chat options on a website you may be browsing to make a purchase. We can all appreciate the need to send a quick reminder to a single contact or within group chat - it is an effective way of ensuring the right information gets to the right people, quickly without the need for yet more admin tasks.

For the team at Dentally, developing an instant messaging system within the software that can help dental practitioners and their teams to easily chat to one another, just seemed like a simple way of helping dental practices run smoothly, save time and make better decisions. The product development is part of Dentally's ongoing commitment to innovation to reduce the administrative burden on dental practices,

\section{Biofilm management}

Lunos, from Durr Dental, is a premium air polishing brand incorporating one powder jet handpiece and two interchangeable nozzles and a range of prophylaxis powders to assist in the minimally invasive management of biofilm.

The powder jet handpiece, called MyLunos, comes with both a supragingival and a subgingival nozzle. Both nozzles can be rotated 360 degrees, to facilitate access to all areas of the oral cavity. The subgingival nozzle features a clip-on, calibrated, sterile, single use tip for precision subgingival application.

MyLunos also incorporates the unique exchangeable powder chamber principle, which means the powder container can be replaced quickly and easily mid treatment, moving from

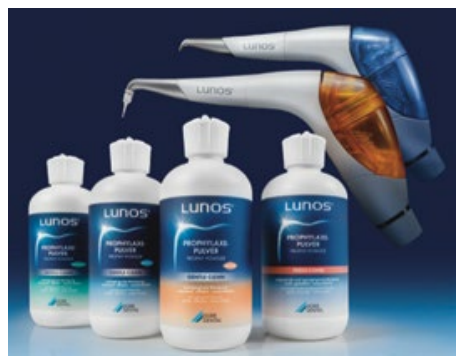
the supra grade powder to sub grade powder with minimal inconvenience and avoiding the need to refill chambers in the middle of treatment. Furthermore, surgeries can prepare enough powder containers for the whole day. All components are autoclavable and the handpieces are available in five different fittings, allowing use on any standard turbine coupling.

MyLunos works with various prophylaxis powders. The Gentle Clean variant of Lunos contains innovative new abrasive agents based on the non-carcinogenic disaccharide trehalose, for gentle cleaning in the supragingival area and is available in three different flavours. Alternatively, there's Lunos prophy powder Perio Combi, which can be used for both supragingival and subgingival treatments. The excellent water solubility of this powder enables safe, virtually residue-free dissolution in the periodontal pocket and suction system. Thanks to this, patients no longer experience the unpleasant grittiness typically associated with this type of product and it doesn't clog up the suction unit or the amalgam separator! that it recently reinforced following the publication of the White Paper: The state of dentistry 2019.

As you would expect from Dentally, it is secure and easy to use. The new chat function means that whether you need to have a discreet conversation about a patient without verbalising sensitive information across the practice, simply pass general information or make a request from the surgery to reception team - you can easily have a focused chat through the messaging service. The impact of this for a practice should not only save valuable admin time but improve efficiencies and productivity among team members.

If you would like more information on the chat functionality, then just email hello@dentally.co. Remember over 7,000 dental professionals are already working smarter, working remotely and working securely with Dentally, so why not book your demo today?

\section{One small change}

Pärla Toothpaste Tabs offer an ethical, plastic-free solution to conventional toothpaste.

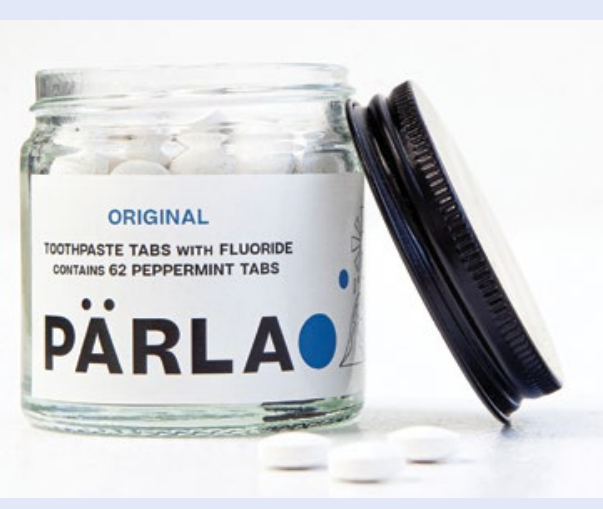

The dehydrated tabs come in a reusable glass jar - to use they are simply chewed for five seconds and then used for brushing as normal with a wet toothbrush. Developed by practising dentists - Simon Chard, Rhona Eskander and Adarsh Thanki - you can rest assured that the product does not compromise on oral health benefits either. SLS-free for reduced risk of irritation, Pärla contains fluoride to protect against decay.

Pärla Toothpaste Tabs are:

- Plastic-free

- Animal cruelty-free

- Palm oil-free

- SLS-free

- Gluten-free

- Made in the UK

- Vegan

- Contain fluoride.

Help your patients make one small change to help protect the planet while maintaining their oral hygiene with innovative Pärla Toothpaste Tabs.

For more information, visit parlatoothpastetabs.com. 